\title{
Performance Evaluation of Wavelength Band Switching in Multi-fiber All-Optical Networks
}

\author{
Xiaojun Cao Vishal Anand Yizhi Xiong Chunming Qiao \\ Department of Computer Science and Engineering \\ State University of New York at Buffalo \\ $\{$ xcao2, vanand, yxiong, qiao $\} @$ cse.buffalo.edu
}

\begin{abstract}
Wavelength band switching (WBS) has only recently attracted attention from the optical networking industry for its practical importance in reducing the control complexity and cost of optical cross-connects (OXCs). However, WBS-related problems of theoretical interest have not been addressed thoroughly by the research community, and many issues are still wide open. In particular, WBS is different from wavelength routing, and thus techniques developed for wavelength-routed networks (including e.g., those for traffic grooming) cannot be directly applied to effectively address WBS-related problems.

In this paper, we first propose a new multi-granular OXC (MG-OXC) architecture for WBS, which is more flexible than any existing WBS node architectures. We also adopt the most powerful waveband assignment strategy, and develop an efficient heuristic algorithm called Balanced Path routing with HeavyTraffic first (BPHT). To verify its near-optimality, we also develop an integer linear programming (ILP) model. Both the ILP and the BPHT algorithms can handle the case with multiple fibers per link and hence are more general than our previous single-fiber solutions [1].

We conduct a comprehensive evaluation of the benefits of WBS through detailed analysis and simulations. We show that the proposed heuristic BPHT can perform much better than a heuristic which applies the optimal routing and wavelength assignment (RWA) method. We also show that WBS using BPHT is even more beneficial in multi-fiber networks than in single-fiber networks in terms of reducing the port count. Our analytical and simulation results also provide valuable insights into the effect of wavelength band granularity, as well as the trade-offs between the wavelength-hop and the port count required in WBS networks.
\end{abstract}

\section{INTRODUCTION}

All-optical networks using wavelength-divisionmultiplexing (WDM) have become promising candidates for the Internet backbone. The WDM technology divides the enormous fiber bandwidth into a large number of wavelengths and with current technologies, each fiber can have 100 or more wavelengths (each operating at $2.5 \mathrm{Gbps}$ or higher). However, such advances in the transmission technology also bring about tremendous increase in the number of ports (and thus the cost) at optical cross-connects (OXCs) as well as the complexity and difficulty associated with control and management of such large scale OXCs.

Recently, the concept of Wavelength Band Switching (WBS) or simply wavebanding has been proposed by several optical networking companies to maintain this complexity at a reasonable level. The main idea of WBS is to group several wavelengths together as a band and switch the band using a single port whenever possible. Systems having a high wavelength count can use a hierarchical or multi-granular $\mathrm{OXC}$ (MG-OXC) such as the one proposed in [2]-[4]. Merits of the MG-OXC such as small-scale modularity, cross-talk and complexity reduction were summarized in [2]. The concept of WBS was applied to WDM ring networks in [5]-[7], and a three-layer MG-OXC architecture for mesh network and its application to metro-area networks were described in [3], [8] while a single-layer architecture was proposed in [9]. Research in efficient WBS algorithm design and their performance evaluation, as well as other problems of more theoretical interest has only begun in academia [1], [10], and many WBS-related problems are still wide open.

In this paper, we first propose a new MG-OXC architecture (shown in Figure 1), which is more flexible than any previously proposed architectures including those in [1], [3]. This architecture allows dynamic configuration of the add, drop and bypass ports, while the earlier architectures only allow fixed add, drop and bypass. The rational behind using such a MGOXC to reduce the port count is that a fiber is demultiplexed into bands if and only if necessary (for example, it carries at least one lightpath which needs to be dropped or added). Similarly, a band is demultiplexed into individual wavelengths if and only if necessary. On the other hand, the MG-OXC with only a wavelength cross-connect (WXC) layer becomes what will be called an ordinary-OXC (single-granular).

WBS differs from conventional wavelength routing in several ways, for example, each has different objectives. Accordingly, techniques developed for wavelength-routed networks (including e.g., those for traffic grooming) cannot be directly applied to effectively address WBS-related problems. More specifically, in networks employing ordinary-OXC, the routing and wavelength assignment (RWA) problem is to find a route for a lightpath and assign a wavelength to it. One of the key objectives of the traditional RWA algorithms is to minimize the total number of wavelength-hop (WH) or the maximum number of wavelengths required to satisfy a given set of lightpath requests, which is known to be NP-complete [11][13]. In this paper, we study the optimal WBS problem, with its main objective being to route lightpaths and assign appropriate wavelengths to them so as to minimize the total number of ports required by the MG-OXCs. As to be shown, even though traditional RWA is still an important component of WBS, new waveband assignment algorithms need to be 
developed in order to effectively achieve the objective.

The above optimal WBS problem is still an open and challenging problem. Recently, an integer linear programming (ILP) model was proposed in [10], but the model is restrictive in that it tries to band or group lightpaths with the same destination only. Further, it requires wavelength conversion capability at $W X C$ layer. In this paper, we adopt a more general, and in fact the most powerful waveband assignment strategy that can group lightpaths with different sources and different destinations when developing an ILP model.

Since the optimal WBS problem contains an instance of RWA, which is NP-complete, it can be solved for a small problem size (e.g. network size) only. Accordingly, we also develop and compare heuristic algorithms for large problem sizes. We show that for small networks, the proposed waveband assignment heuristic, called Balanced Path routing with Heavy-Traffic first (or BPHT), can achieve results that are close to the optimal results, and can significantly outperform a heuristic based on the optimal RWA algorithm. We develop an analytical model to calculate the number of ports needed and in particular, analyze the performance of the BPHT heuristic, which is verified through extensive simulation. To the best of our knowledge, this is the first comprehensive performance evaluation study on optimal WBS and efficient WBS heuristics for mesh networks.

Note that the ILP formulation, and the BPHT heuristic developed in this paper are applicable to multi-fiber networks, and hence are more general than our previous single-fiber solutions presented in [1]. The study on multi-fiber networks is motivated by the work in [14]-[17], which has shown that the performance improvement in terms of reduced blocking and better fault tolerance can be obtained by using multifiber networks. Ref. [14] showed that doubling the number of fibers per link is akin to doubling the number of wavelengths per link with the additional advantage of simulating a partial wavelength conversion capability. In this paper, we analyze the performance advantages of using multi-fiber links over singlefiber links in WBS networks and show that significant savings in number of ports can be achieved in multi-fiber networks.

Since the objective of WBS is to minimize the number of ports in MG-OXCs, rather than to minimize WHs as in traditional RWA algorithm, WBS may require more WHs than that needed by optimal RWA to satisfy a given set of lightpaths requests. In other words, there is a trade-off between minimizing the number of WHs versus minimizing the number of ports in WBS networks. Our results indicate that our heuristic BPHT achieves a large reduction in the number of required ports with only a small increase in WHs.

This paper is organized as follows. In Section II, We describe the proposed WBS node architecture and explain how WBS is performed using this flexible MG-OXC. Section III presents our ILP model while Section IV presents heuristic algorithms including the proposed BPHT heuristic. In Section V, we analyze the performance of BPHT. Numerical results from our ILP model and heuristic algorithms are presented in Section VI. Finally, Section VII concludes the paper with a summary of its major contributions.

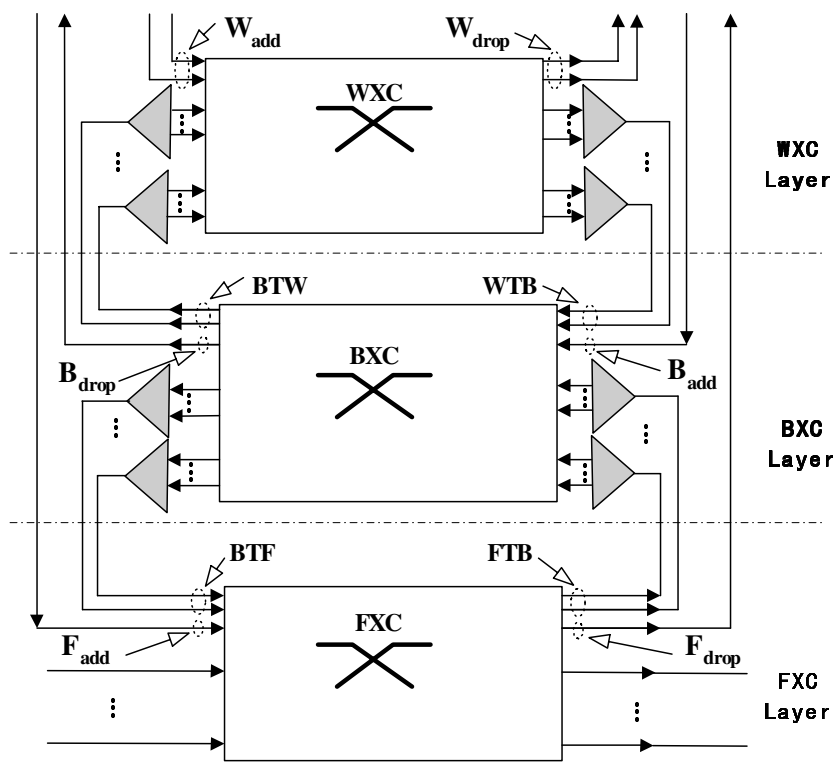

Fig. 1. Architecture of an MG-OXC

\section{WAVELENGTH BAND SWITCHING}

In this section, we describe the proposed, flexible MGOXC architecture shown in Figure 1, which includes the $F X C, B X C$ and $W X C$ layers. As shown in the figure, the $W X C$ and $B X C$ layers consist of cross-connect(s) and multiplexer(s)/demultiplexer(s). The WXC layer includes a wavelength cross-connect (WXC) switch that is used to bypass/add/drop lightpaths at this layer, band-to-wavelength (BTW) demultiplexers, and wavelength-to-band (WTB) multiplexers. The BTW demultiplexers are used to demultiplex bands into wavelengths, while the WTB multiplexers are used to multiplex wavelengths into bands. At the $B X C$ layer, the waveband cross-connect (BXC) switch is used to bypass/add/drop wavebands. The $B X C$ layer also includes the fiber-to-band (FTB) demultiplexers and band-to-fiber (BTF) multiplexers. Similarly, fiber cross-connect (FXC) switch is used to bypass/add/drop fibers at the $F X C$ layer.

The MG-OXC architecture proposed in this paper is different and more flexible than any existing architectures including those considered in [1], [3], as it allows for dynamic interconnection or configuration of the MG-OXC. For example, at the $F X C$ layer, as long as there is a free $F T B$ port, any fiber can be demultiplexed into bands. Similarly, at the $B X C$ layer any band can be demultiplexed to wavelengths using a free $B T W$ port by appropriately configuring the $\mathrm{FXC}, \mathrm{BXC}$ crossconnects and associated demultiplexers. On the other hand, in the earlier proposed architectures, these configurations are fixed, in that only certain fixed fibers (bands) can be demultiplexed. Due to the difference between this new architecture and the one considered in [1], the way to count the number of ports are also different. 
More specifically, when counting the number of ports, we will only focus on the input-side of the MG-OXC ${ }^{1}$. We define the input-side of a MG-OXC to consist of locally added traffic and traffic coming into the MG-OXC node from all other nodes (which consists of bypass traffic and locally dropped traffic). In order to reduce the number of ports, the MGOXC switches a fiber using one port (space switching) at the FXC cross-connect if none of its wavelengths is used to add or drop a lightpath. Otherwise, it will demultiplex the fiber into bands, and switch an entire band using one port at the BXC cross-connect if none of its wavelengths is used to add or drop a lightpath. In other words, only the band(s) whose individual wavelengths need to be added or dropped will be demultiplexed, and only the wavelengths in those bands that carry bypass traffic need to be switched using the WXC. This is in contrast to the ordinary-OXCs, which needs to switch every wavelength individually using one port. For example, assume there are 10 fibers, each having 100 wavelengths, and one wavelength needs to be dropped and one to be added at a node. The total number of ports required at the node when using an ordinary-OXC is 1000 for incoming wavelengths (including 999 for bypass and 1 for drop wavelength), plus 1 for add wavelength for a total of 1001 . However, if the 100 wavelengths in each fiber are grouped into 20 bands, each having 5 wavelengths, then using a MG-OXC, only one fiber needs to be demultiplexed into 20 bands (using a 11-port FXC). Then, only one of these 20 bands needs to be demultiplexed into 5 wavelengths (using a 21-port BXC). Finally, one wavelength is dropped and added (using a 6-port WXC). Accordingly, the MG-OXC has only $11+21+6=38$ ports, an almost 30 times reduction.

Hereafter, we concentrate on one of the proposed WBS schemes in [1], wherein each fiber has a fixed number $(\mathcal{B})$ bands and each band has a fixed number $(\mathcal{W})$ as well as a fixed set of wavelengths. Note that the ILP model and heuristic algorithms developed in this paper can be extended to the other WBS schemes (e.g., allowing variable number of bands per fiber) as well. With the current state of art, wavelength conversion technology is still too immature (and expensive), hence, in the following, we assume that there is no wavelength conversion in our model. The case with wavelength conversion and other variations of the WBS scheme will be studied in the future.

\section{ILP MODEL FOR WBS}

This section formulates the WBS scheme using integer linear programming (ILP). The ILP formulation is for multifiber networks, and is more general than existing ILP models, including that for the single-fiber case in [1].

\footnotetext{
${ }^{1}$ Due to the symmetry of the MG-OXC architecture, the number of ports on the input-side and output-side are equal.
}

\section{A. Notations}

$I_{n}: \quad$ Set of input fibers at node $n$ (excluding those for local add);

$\mathcal{I}_{n, m}^{f}: \quad$ Input fiber $f$ at node $n$, connected to node $m$. So $I_{n}=\bigcup_{m, f} \mathcal{I}_{n, m}^{f}$;

$O_{n}: \quad$ Set of output fibers at node $n$ (excluding those for local drop);

$\mathcal{O}_{n, m}^{f}: \quad$ Output fiber $f$ at node $n$, connected to node $m$. So $O_{n}=\bigcup_{m, f} \mathcal{O}_{n, m}^{f}$;

$A_{n}: \quad$ Set of local add fibers at node $n$, including those used at the ports of WXC, BXC and FXC layer;

$D_{n}: \quad$ Set of local drop fibers at node $n$, including those used at the ports of WXC, BXC and FXC layer;

$I A_{n}: \quad I_{n} \bigcup A_{n}$. This set includes the set of all incoming fibers (local and non-local) at node $n$;

$O D_{n}: \quad O_{n} \bigcup D_{n}$. This set includes the set of all outgoing fibers (local and non-local) at node $n$;

$£_{b}$ : $\quad$ Set of wavelengths in band $b$;

$\mathcal{F}$ : $\quad$ Number of fibers per link that can be used for each direction;

$\mathcal{K}: \quad$ Number of wavelengths per fiber;

$\mathcal{B}: \quad$ Number of wavelength bands per fiber;

$\mathcal{W}$ : Number of wavelengths per wavelength band $(\mathcal{K}=\mathcal{B} \times \mathcal{W})$

P: $\quad$ Set of node pairs having non-zero traffic demand. Each node pair can be denoted by $p=$ (p.src,p.dest), where p.src and p.dest represent the source and destination nodes of one or more request lightpaths, respectively;

$\mathrm{T}[\mathrm{p}]: \quad$ Traffic matrix whose element $t_{p}$ is an integer, representing the traffic demand (i.e. number of lightpaths) of the node pair $p$.

\section{B. ILP Variables}

To facilitate presentation and understanding of our ILP model, we define variables to describe the properties of a node (instead of a link as in other ILP formulations for RWA). More specifically, to obtain and represent the detailed information of the routing and wavelength assignment, we introduce the following binary variables to be used in the ILP formulation.

Note that the traffic at a node can be drop traffic, bypass traffic or add traffic. The following four variables: $V_{i, o, p}^{n, w}$, $W_{i, o}^{n, w}, B_{i, o}^{n, b}$ and $F_{i, o}^{n}$ are used for describing the lightpaths, each of which can represent bypass traffic when $i \in I_{n}, o \in$ $O_{n}$; add traffic when $i \in A_{n}, o \in O_{n}$ or drop traffic when $i \in I_{n}, o \in D_{n}$. An incoming (or outgoing) fiber refers to either an input (or output) fiber from (or to) a neighboring node or a fiber connecting the local node to any add (or drop) port at the WXC, BXC and FXC layer (as mentioned earlier in Section III-A). 
$V_{i, o, p}^{n, w}: \quad 1$ if node $n$ has a lightpath for node pair $p=$ (p.src, p.dest) on wavelength $w$ from incoming fiber $i$ to outgoing fiber $o$, and 0 otherwise;

$W_{i, o}^{n, w}: \quad 1$ if node $n$ has a wavelength $w$ bypass/add/drop at the WXC layer from incoming fiber $i$ to outgoing fiber $o$, and 0 otherwise;

$B_{i, o}^{n, b}: \quad 1$ if node $n$ has a waveband $b(b \in[1,2, \ldots, \mathcal{B}])$ bypass/add/drop at the $B X C$ layer from incoming fiber $i$ to outgoing fiber $o$, and 0 otherwise;

$F_{i, o}^{n}: \quad 1$ if node $n$ has an incoming fiber $i$ bypass/add/drop to outgoing fiber $o$ at the $F X C$ layer, and 0 otherwise;

Four additional variables used for describing multiplexing/demultiplexing are also defined.

$F T B_{i}^{n}: \quad 1$ if input fiber $i\left(i \in I_{n}\right)$ needs to be demultiplexed into bands at node $n$, and 0 otherwise;

$B T W_{i}^{n, b}: \quad 1$ if band $b$ on input fiber $i\left(i \in I_{n}\right)$ needs to be demultiplexed into wavelengths at node $n$, and 0 otherwise;

$B T F_{o}^{n}$ : $\quad 1$ if a band needs to be multiplexed onto an output fiber $o\left(o \in O_{n}\right)$ at node $n$, and 0 otherwise;

$W T B_{o}^{n, b}: \quad 1$ if a wavelength needs to be multiplexed on to band $b$ of an output fiber $o\left(o \in O_{n}\right)$ at node $n$, and 0 otherwise;

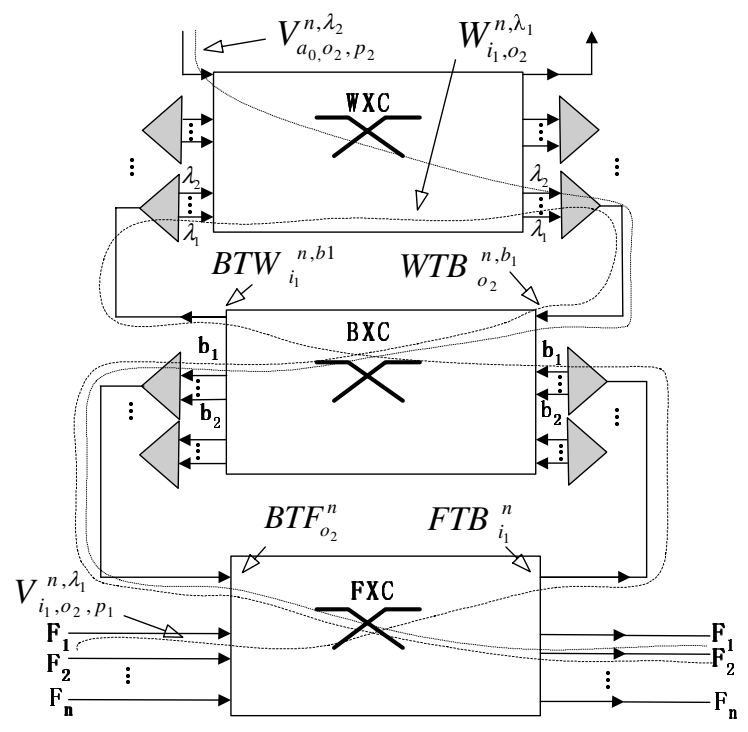

Fig. 2. Waveband at node $n$

As a consequence of multiplexing/demultiplexing, we need to use multiplexer/demultiplexer port(s) at the respective layers. Figure 2 shows one such example involving two lightpaths, one for node pair $p_{1}$ using $\lambda_{1}$ on input fiber 1 and the other for node pair $p_{2}$ using $\lambda_{2}$ to be added locally. Using the MG$\mathrm{OXC}$, the two lightpaths are grouped together in the same band of the same output fiber (e.g. fiber 2). By definition, we have $V_{i_{1}, o_{2}, p_{1}}^{n, \lambda_{1}}=V_{a_{0}, o_{2}, p_{2}}^{n, \lambda_{2}}=1$. For this, input fiber 1 (containing the lightpath for $p_{1}$ ) has to be demultiplexed into band $b_{1}$ (and other bands) using a FTB demultiplexer (hence, $F T B_{i_{1}}^{n}=1$ ).
Band $b_{1}$ then has to be further demultiplexed into $\lambda_{1}$ and other wavelengths (hence, $B T W_{i_{1}}^{n, b_{1}}=1$ ) to switch the lightpath for $p_{1}$ (hence, $W_{i_{1}, o_{2}}^{n, \lambda_{1}}=1$ ). The second lightpath for $p_{2}$ is added into band $b_{1}$ using a WTB multiplexer (hence, $W T B_{o_{2}}^{n, b_{1}}=1$ ). Now that the two lightpaths are in the same band, the band is multiplexed onto a fiber using a BTF multiplexer (hence, $B T F_{o_{2}}^{n}=1$ ), and then transmitted onto output fiber 2 .

\section{Objective Function}

Let $W X C_{n}, B X C_{n}$ and $F X C_{n}$ be the number of ports at $W X C, B X C$ and $F X C$ layers at node $n$, respectively. There are two reasonable objectives. The first is to minimize the total cost associated with the MG-OXC ports in the network, that is,

$$
\text { minimize }\left[\alpha \times \sum_{n} W X C_{n}+\beta \times \sum_{n} B X C_{n}+\gamma \times \sum_{n} F X C_{n}\right]
$$

where $\alpha, \beta$ and $\gamma$ are the coefficients or weights corresponding to the cost of each port at the $W X C, B X C$ and $F X C$ layer, respectively. When $\alpha=\beta=\gamma=1$, the objective becomes to minimize the total number of MG-OXC ports in the network, which is the sum of the port count at FXC, BXC and WXC layers respectively.

The second objective is to minimize the maximum cost at each node over all nodes. This can be formulated as:

$$
\text { minimize } \max \left(\alpha \times W X C_{n}+\beta \times B X C_{n}+\gamma \times F X C_{n}\right)
$$

When $\alpha=\beta=\gamma=1$, this becomes equal to minimizing the maximum port count (node size) over all the nodes in the network.

\section{Constraints}

For Routing and Wavelength Assignment, the following constraints on traffic flows, wavelength-capacity and wavelength-continuity are similar to those in the traditional RWA ILP formulations.

$$
\begin{aligned}
& \begin{cases}\sum_{i \in A_{n}, o \in O_{n}} V_{i, o, p}^{n, w}=\sum_{i \in I_{n}, o \in D_{n}} V_{i, o, p}^{n, w}=0 & n \neq p . s r c, p . \operatorname{des} t, \forall w \\
\sum_{w, i \in A_{n}, o \in O_{n}} V_{i, o, p}^{n, w}=t_{p} & n=p . s r c, \\
\sum_{w, i \in I_{n}, o \in D_{n}} V_{i, o, p}^{n, w}=t_{p} & n=p . \text { dest }\end{cases} \\
& \sum_{p, o \in O D_{n}} V_{i, o, p}^{n, w} \leq 1 \quad \forall w, i \in I_{n} \\
& \sum_{p, i \in I A_{n}} V_{i, o, p}^{n, w} \leq 1 \quad \forall w, o \in O_{n} \\
& \sum_{i \in I A_{m}, o \in \mathcal{O}_{m, n}^{f}} V_{i, o, p}^{m, w}-\sum_{i \in \mathcal{I}_{n, m}^{f}, o \in O D_{n}} V_{i, o, p}^{n, w}=0 \quad \forall m, n, p, w, f ;
\end{aligned}
$$

Equation (3) is the traffic flow constraint; Equations (4) and (5) are the wavelength capacity constraint; Equation (6) is the wavelength continuity constraint. 
For Waveband Switching, we need the following additional constraints.

$$
\begin{gathered}
1 \geq F_{i, o}^{n}+B_{i, o}^{n, b}+W_{i, o}^{n, w} \geq \sum_{p} V_{i, o, p}^{n, w} \forall w \in £_{b}, i \in I A_{n}, o \in O D_{n} ; \text { (7) } \\
1 \geq F_{i, o}^{n}+\sum_{p, o_{1} \neq o} V_{i, o_{1}, p}^{n, w}, \quad 1 \geq F_{i, o}^{n}+\sum_{p, i_{1} \neq i} V_{i_{1}, o, p}^{n, w} \forall w, i, o ; \quad \text { (8) } \\
1 \geq B_{i, o}^{n, b}+\sum_{p, o_{1} \neq o} V_{i, o_{1}, p}^{n, w}, \quad 1 \geq B_{i, o}^{n, b}+\sum_{p, i_{1} \neq i} V_{i_{1}, o, p}^{n, w} \forall i, o, w \in £_{b} ; \text { (9) }
\end{gathered}
$$

Constraints (7), (8) and (9) ensure that if a lightpath uses wavelength $w$ belonging to band $b$ of incoming fiber $i$ and outgoing fiber $o$ (i.e. $\sum_{p} V_{i, o, p}^{n, w}=1$ ), then at node $n$,

- exactly one of FXC, BXC and WXC cross-connect port will be used for switching this lightpath when it is a bypass (i.e. $i \in I_{n}, o \in O_{n}$ ) or

- exactly one of $F_{a d d}, B_{a d d}$ and $W_{a d d}$ port will be used for adding this lightpath when it is added (i.e. $i \in$ $A_{n}, o \in O_{n}$ ) or

- exactly one of $F_{d r o p}, B_{d r o p}$ and $W_{d r o p}$ port will be used for dropping this lightpath when it is dropped (i.e. $\left.i \in I_{n}, o \in D_{n}\right)$.

$$
B T F_{o}^{n} \geq W T B_{o}^{n, b} \geq W_{i, o}^{n, w} \forall w \in £_{b}, o \in O_{n}, i \in I A_{n} ;
$$

The above constraint ensures that a wavelength $w$ at node $n$ switched or added at the $W X C$ layer has to pass a WTB multiplexer to the $B X C$ layer. At the same time, every band from a WTB multiplexer has to pass a BTF multiplexer before it can leave node $n$. Similarly, Equation (11) below specifies that a wavelength $w$ switched or dropped at the WXC layer has to come from $B X C$ layer using a BTW demultiplexer, and in addition every band demultiplexed by BTW can only come from a FTB demultiplexer.

$$
F T B_{i}^{n} \geq B T W_{i}^{n, b} \geq W_{i, o}^{n, w} \forall w \in £_{b}, o \in O D_{n}, i \in I_{n} ;
$$

Finally, any bypass or add bands should pass a BTF multiplexer as specified in equation (12) and similarly, any drop or bypass band can only come from a FTB demultiplexer as specified in Equation (13) .

$$
\begin{aligned}
& B T F_{o}^{n} \geq B_{i, o}^{n, b} \forall o \in O_{n}, i \in I A_{n} ; \\
& F T B_{i}^{n} \geq B_{i, o}^{n, b} \forall o \in O D_{n}, i \in I_{n} ;
\end{aligned}
$$

For Port Numbers, the following constraints specify the minimum number of ports required at each layer of the MGOXC.

$$
\begin{gathered}
W X C_{n}=\sum_{i \in I A_{n}, o \in O D_{n}, w} W_{i, o}^{n, w} \forall n ; \\
B X C_{n}=\sum_{i \in I A_{n}, o \in O D_{n}, b} B_{i, o}^{n, b}+\sum_{o \in O_{n}, b} W T B_{o}^{n, b}+\sum_{i \in I_{n}, b} B T W_{i}^{n, b} \forall n ; \\
F X C_{n}=\sum_{i \in I A_{n}, o \in O D_{n}} F_{i, o}^{n}+\sum_{o \in O_{n}} B T F_{o}^{n}+\sum_{i \in I_{n}} F T B_{i}^{n} \forall n ;
\end{gathered}
$$

For the $W X C$ layer, the number of input-side ports include the bypass, add/drop lightpaths as specified in (14). The number of input-side ports needed at the $B X C$ layer is the sum of the number of wavebands $B_{i, o}^{n, b}$ (BXC cross-connect and add/drop/bypass bands) and the number of wavebands from the WTB/BTW multiplexers/demultiplexers as in (15). Similarly, Equation (16) can be used to determine the number of ports at the $F X C$ layer.

In short, our ILP model (and heuristics to be described next) considers the design of MG-OXC nodes (i.e. the number of ports allocated at each of the layers) with the objective to minimize either the total port count or the maximum port count over all MG-OXC nodes in the network given a set of traffic demands to be satisfied on a given network topology, wherein each link in the network may have single or multiple fibers.

Note that if we eliminate the $F X C$ and $B X C$ layers (i.e. set corresponding variables to 0 ) from the $\mathrm{MG}-\mathrm{OXC}$, the above ILP formulation with Objective (1) will minimize the total number of ports, which is equivalent to minimizing WHs using ILP for optimal RWA. As such ILP formulations developed can only be solved for small systems with a few nodes and a few wavelengths on each fiber, we need to develop efficient heuristic-based approaches for large systems.

\section{Heuristic Algorithms for Waveband Switching}

In this section, we describe the heuristic algorithms developed for WBS. There are several waveband assignment strategies in WBS networks, including: (1) grouping the lightpaths with the same source-destination pair only; (2) grouping the lightpaths from the same source only; (3) grouping the lightpaths with same destination only; (4) grouping the lightpaths with common intermediate links (from any source to any destination). The authors in [10] only considered the Strategy 3 and single-fiber networks, while our ILP formulation covers the fourth strategy, which is the most generic and flexible in multi-fiber networks. Below, we describe a heuristic that takes into consideration lightpath routing as well as waveband assignment Strategy 4 in multi-fiber networks.

\section{A. Waveband Oblivious Optimal RWA (WBO-RWA)}

To study the relationship between WBS and traditional RWA, we use ILP formulations for RWA [12] that minimize the total number of used WHs. Then we try to group the assigned wavelengths into bands and calculate the number of required ports. Note that the heuristic is completely oblivious to the existence of wavebands. From now on, we refer WBORWA as getting wavelength assignment from the optimal RWA ILP formulation and then grouping them [1]. 


\section{B. Balanced Path routing with Heavy-Traffic first waveband assignment (BPHT)}

Intuitively, to maintain wavelength-continuity in wavelength routed optical networks without wavelength conversion, it is better to assign wavelengths to longer paths (in terms of hops) first. Further, to reduce the number of ports in MG-OXC, it is better to assign paths that have maximum number of links in common, wavelengths in the same fiber (and band), thus increasing the probability of switching the whole fiber (and band) by just using a single FXC (and BXC) port. The following is our three-stage heuristic algorithm called Balanced Path routing with Heavy-Traffic (BPHT) first waveband assignment, which tries to maximize the reduction in the MG-OXC size using the above ideas.

\section{Stage 1: Balanced Path Routing}

In this stage, we use the following steps to achieve load balanced routing.

- Find K-shortest routes for every node pair $(s, d)$ with non-zero traffic demand as in [18], and order them from the shortest to the longest (in terms of hop number) as $P_{s, d}^{1}, P_{s, d}^{2}, \cdots, P_{s, d}^{k}$. Let the number of hops of the shortest route be $H_{s, d}$.

- Define the load on every link $l$ to be the number of routes already using link $l$ (initially, this is 0 ). Let $C$ be the maximum link load over all the links.

- Use $C$ to achieve load balanced routing, starting with the node pair $(s, d)$ with the largest $H_{s, d}$ value over all node pairs, to determine the route for each node pair. More specifically, for the K-shortest routes $P_{s, d}^{i}$ of the selected node pair $(s, d)$, where $i=1,2, \cdots, k$, we compute the $C$ and pick one of the routes that minimizes $C$. If more than one routes, say $P_{s, d}^{i}$ and $P_{s, d}^{j}$, have the same minimum $C$, the shortest one (i.e. $P_{s, d}^{i}$, if $i<j$ ) will be used as the route for $(s, d)$. That is, all the lightpaths from $s$ to $d$ will take this route. After the route for $(s, d)$ is chosen, the process continues to choose one route for each of the remaining node pairs, starting with the one having the largest number of hops along the shortest path, until every node pair with non-zero traffic demand is assigned a route.

\section{Stage 2: Wavelength Assignment}

Based on the observation that bypass traffic, which goes through two or more hops accounts for $60 \%-80 \%$ of the total traffic in the backbone, we assign the wavelengths to those bypass lightpaths first. At the same time, we also want to give preference to the lightpaths that overlap with many other (shorter) lightpaths in order to maximize the advantage of wavebanding.

The following steps are used to assign wavelengths to all the lightpath demands once the routing is done in Stage 1. To maximize the benefit of WBS in multi-fiber networks, we introduce a new waveband assignment algorithm, called waveband assignment for multi-fiber WBS (WA-MF-WBS, see Step (D) below).
(A) For every node pair $(s, d)$, whose route is determined as $s=s_{0} \rightarrow s_{1} \rightarrow s_{2} \ldots s_{n-1} \rightarrow s_{n}=d$ in Stage 1, define a set $Q_{d}^{s}$, which includes all node pairs $\left(s_{i}, s_{j}\right)$, whose route is $s_{i}, s_{i+1}, \ldots, s_{j}$, as determined in Stage 1 , where $0 \leq i \leq n-2$, and $i+2 \leq j \leq n$. Note that it is possible that the route chosen for $\left(s_{i}, s_{j}\right)$ in Stage 1 is not a sub-path of the route chosen for $(s, d)$, in which case, $\left(s_{i}, s_{j}\right)$ will not belong to $Q_{d}^{s}$.

(B) Calculate the weight (similar to the concept of wavelength-hops) for each set $Q_{d}^{s}$ as $W_{s d}=$ $\sum_{p \in Q_{d}^{s}} h_{p} \times t_{p}$, where $p=\left(s_{i}, s_{j}\right) \in Q_{d}^{s}, h_{p}$ is the number of hops and $t_{p}$ is the required number of lightpaths from $s_{i}$ to $s_{j}$;

(C) Find the set $Q_{d}^{s}$ with the largest $W_{s d}$.

(D) Call set $Q_{d}^{s}$ as $\mathcal{L}$, and assign wavelengths to $\mathcal{L}$ as follows. i.) Suppose that the longest path in $\mathcal{L}$ is as follows: $s_{0} \rightarrow s_{1} \rightarrow s_{2} \ldots s_{n-1} \rightarrow s_{n}$. Let $s=s_{0}$ and $d=s_{n}$ (which is the case initially based on the definition of $\left.Q_{d}^{s}\right)$. Assign wavelengths to the requested lightpaths for the node pair $(s, d)$ by trying to group them into the same fiber, and within each fiber, into the same band(s). More specifically, for each fiber, let $0 \leq w \leq \mathcal{K}-1$ and $0 \leq b \leq \mathcal{B}-1$ be the index of wavelength and band respectively, starting from which, an available wavelength and band will be searched in order to fulfill new lightpath requests; In addition, let $0 \leq f \leq \mathcal{F}-1$ be the index of the fiber currently under consideration (i.e., whose wavelengths may be used for new lightpaths). Initially, $f=0$ and $w=b=0$ for all fibers. The following algorithm $W A-M F-W B S$ assigns wavelengths to the lightpaths for a specified node pair $p$.

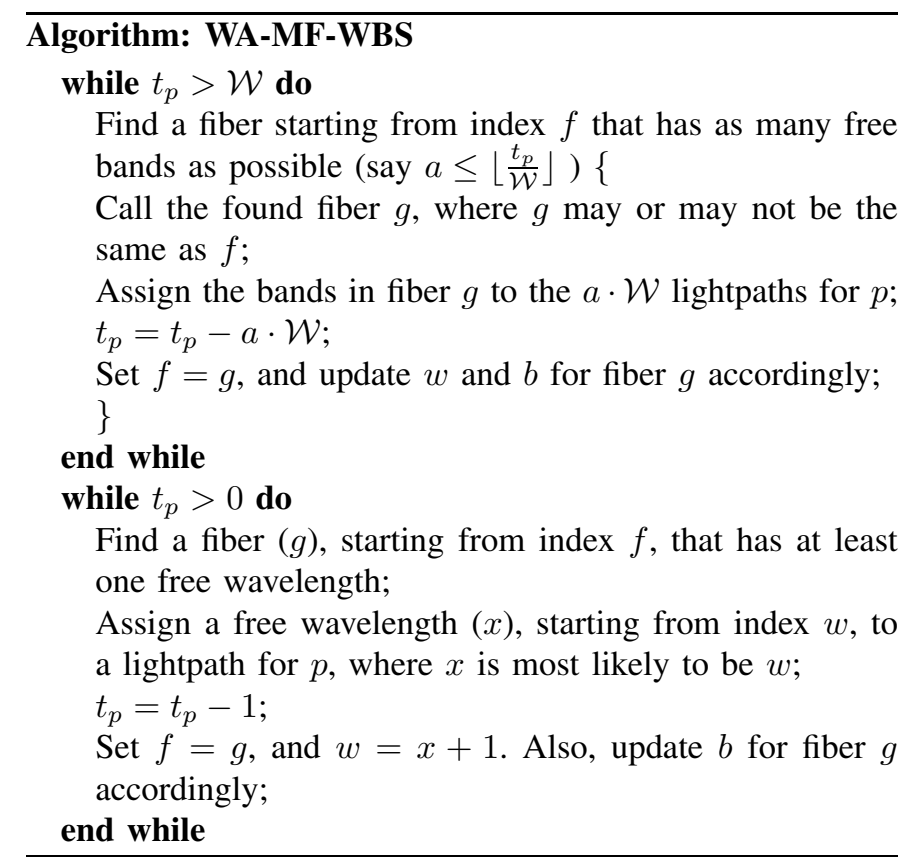

ii.) Use $W A-M F-W B S$ to assign wavelengths to the 
requested lightpaths for $\left(s, s_{j}\right)$ starting with the largest $j$ (i.e. $j=n-1, n-2, \ldots, 2$ ).

iii.) Use $W A-M F-W B S$ to assign wavelengths to the requested lightpaths for $\left(s_{i}, d\right)$ starting with the smallest $i$ (i.e. $i=1,2, \ldots, n-2$ ).

iv.) If there are still node pairs $\left(s_{i}, s_{j}\right) \in Q_{d}^{s}$ that have not been considered, repeat from Step (D) by treating $s_{i}$ with the smallest $i$ as $s$, and $s_{j}$ with the largest $j$ as $d$. Otherwise go to Step (E).

(E) Recompute the weight for those node pairs whose routes use any part of the route used by node pair $(s, d)$. For each fiber, re-adjust $b$ and $w$ to be the "next" waveband and the first wavelength in the next waveband, respectively, so as to prevent the lightpaths of the next node pair set (e.g. $\left.Q \begin{array}{c}s^{\prime} \\ d^{\prime}\end{array}\right)$ from using the same bands as the lightpaths of $Q_{d}^{s}$ (thus reducing the need to demultiplex and multiplex the lightpaths belonging to these two sets when they merge and diverge). More specifically, set $b=(b+1) \bmod \mathcal{B}$, and $w=b \times \mathcal{W}$, and then go to step $(C)$. Repeat until all the bypass (multi-hop) lightpath demands are satisfied.

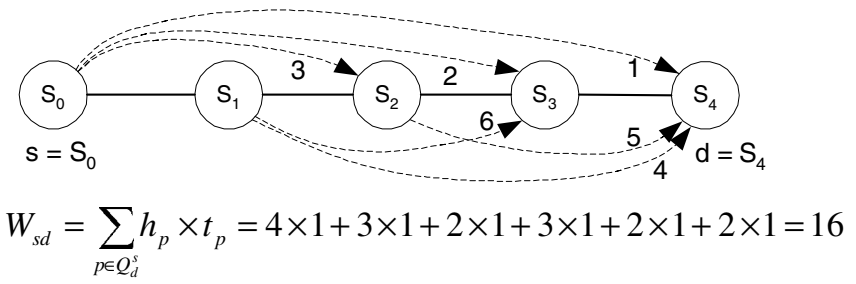

Fig. 3. An example illustrating the Steps (C) and (D) in Stage 2 of BPHT

For example, suppose we are considering a node pair set $Q_{4}^{0}$ in Figure 3, where $t_{p}=1$ for any $p \in Q_{4}^{0}$. Assuming that the lightpaths numbered from 2 to 6 will be routed along the same route as the lightpath 1 ( i.e. $s_{0} \rightarrow s_{1} \rightarrow s_{2} \rightarrow s_{3} \rightarrow s_{4}$ ) as dictated by the load balanced routing algorithm. Then, the weight of the node pair set is 16 as shown, and in addition, the order in which these lightpaths will be assigned wavelengths according to Steps (C) and (D) is from 1 to 6.

(F) Finally, assign wavelengths to lightpaths between two nodes separated by only one hop, starting with the node pair having the largest lightpath demand.

\section{Stage 3: Waveband Switching}

Once the wavelength assignment is done, WBS can be performed in a fairly straight-forward way. Basically, we switch as many fibers using FXCs as possible; and then as many wavebands using BXCs as possible. The remaining lightpaths are then individually switched at the WXC layer. The total number of ports used at a given node can then be determined as discussed at the Section III-D.

Ideally, BPHT will group traffic from the same source to the same destination, and most of the traffic that has common intermediate links. One of the variations of BPHT (in Stage 1 ) is to balance the amount of traffic (in terms of the actual number of lightpaths instead of just one route for each node pair) on every link. Another variation is to assign wavelengths to lightpaths with the largest hop count or those for node pairs with the largest weighted traffic demand (i.e. $h_{p} \times t_{p}$ ) first (assuming e.g., shortest-path routing) in Stage 2. In our experiments, we have compared many heuristics and found that the overall performance of BPHT is the best. Due to space limitation, we will only compare the results of BPHT, with that of WBO-RWA and previous ILP model.

\section{Performance Analysis}

To simplify the analysis of the performance of our heuristic algorithm BPHT, we assume a random network with $N$ nodes and $2 L$ unidirectional links and a uniform traffic model where the traffic demand of every node pair $p$ is $t$ (i.e. $t_{p}=t$ ). Let $\delta=\frac{2 L}{N}$ be the average node degree, $H(p)$ be the shortest path length for node pair $p$, and $G=\left\lceil\frac{\sum_{p \in P} t \times H(p)}{2 L}\right\rceil$ be the average number of lightpaths on a link. Note that the minimum amount of total traffic going into the input-side of all the nodes is $M P=\sum_{p \in P} t \times H(p)+N(N-1) \times t$, where $N(N-1) \times t$ is total number of added lightpaths. Further, note that MP is also equal to the minimum number of ports in ordinary-OXC networks.

Given that the proposed heuristic BPHT uses load balanced routing, it is reasonable to assume that all added (or dropped, bypass) traffic, measured in terms of the number of lightpaths, is evenly distributed among all the output (or input) links. This implies that the number of dropped (D) or added (A) lightpaths on a link at a node is $A=D=\left\lceil\frac{(N-1) \times t}{\delta}\right\rceil$ and the number of bypassing lightpaths on a link is $I=G-D$.

\section{Case 1: Traffic demand (t) is not a multiple of the waveband granularity $(\mathcal{W})$}

If the traffic demand $(\mathrm{t})$ is not a multiple of the waveband granularity, each of the three layers contributes to the total port count of the MG-OXC. Below, we calculate the ports at each of the layers at a node $n$ starting at the FXC layer.

We note that, for a given link, $F_{a}=F_{d}=\left\lfloor\frac{D}{\mathcal{K}}\right\rfloor$ is the number of full fibers that can be added or dropped. On the other hand, $F_{b}=\left\lfloor\frac{I}{\mathcal{K}}\right\rfloor$ is the number of full fibers that will bypass. The remaining number of lightpaths on the link is thus $\lambda_{F T B}=G-F_{d} \times \mathcal{K}-F_{b} \times \mathcal{K}$. Hence, $F T B=\left\lceil\frac{\lambda_{F T B}}{\mathcal{K}}\right\rceil$ is the number of FXC layer ports needed for FTB demultiplexers. Due to the symmetry in added and dropped traffic (i.e. uniform traffic), we have $F T B=B T F$ (and $F_{a}=F_{d}$, as above).

Accordingly, at the FXC layer of node $n$ (having $\delta$ links), the number of required ports include $F_{i}=\left\lceil\frac{G}{\mathcal{K}}\right\rceil$ ports for input fibers (from other nodes), $F_{a}$ ports for locally added fibers and $B T F$ ports for lightpaths from BTF multiplexers on each 
connected link. Thus, we have:

$$
F X C_{n}=\left[F_{i}+F_{a}+B T F\right] \times \delta
$$

From the above analysis, we know that there are $D^{\prime}=D-$ $F_{d} \times \mathcal{K}$ remaining lightpaths (per link) that need to be dropped (through the BXC and WXC layers) and $I^{\prime}=I-F_{b} \times \mathcal{K}$ remaining bypass lightpaths. Hence, $B_{d}=\left\lfloor\frac{D^{\prime}}{\mathcal{W}}\right\rfloor$ is the number of ports for bands to be dropped locally, $B_{b}=\left\lfloor\frac{I^{\prime}}{\mathcal{W}}\right\rfloor$ is the number bypass bands and $B T W=\left\lceil\frac{\lambda_{B T W}}{\mathcal{W}}\right\rceil$ is the number of ports for BTW demultiplexers where $\lambda_{B T W}=\lambda_{F T B}-\left(B_{d}+\right.$ $\left.B_{b}\right) \times \mathcal{W}$

Similarly, the number of ports required at the BXC layer of node $n$ includes $B_{i}=\left\lceil\frac{I^{\prime}+D^{\prime}}{\mathcal{W}}\right\rceil$ ports for input bands, $B_{a}$ $\left(=B_{d}\right)$ ports for locally added bands and WTB (=BTW) ports for bands from WTB multiplexers on each connected link. Thus, we have:

$$
B X C_{n}=\left[B_{i}+B_{a}+W T B\right] \times \delta
$$

Finally, for the entire network, the remaining $M P-\left(F_{b}+\right.$ $\left.F_{d}+F_{a}\right) \times \mathcal{K} \times \delta \times N-\left(B_{b}+B_{d}+B_{a}\right) \times \mathcal{W} \times \delta \times N$ lightpaths will go through the WXC layer. Hence, the number of ports required at the $\mathrm{WXC}$ layer is:

$W X C=M P-\left(F_{b}+F_{d}+F_{a}\right) \times \mathcal{K} \times \delta \times N-\left(B_{b}+B_{d}+B_{a}\right) \times \mathcal{W} \times \delta \times N$ Therefore, from equations (17) - (19), we can obtain the total number of ports in the entire network as:

$$
\text { Total }=\left(F X C_{n}+B X C_{n}\right) \times N+W X C
$$

Case 2: Traffic demand (t) is a multiple of the waveband granularity $(\mathcal{W})$

If the traffic demand per node pair is a multiple of $\mathcal{W}$, the MGOXC will add/drop/bypass bands, not individual wavelengths in order to reduce the number of ports. Thus, all traffic is switched using the FXC and BXC layers only, and the WXC layer is not needed. Accordingly, at the BXC layer, this is similar to having an ordinary-OXC, wherein each port switches a band of wavelengths. Hence, in this case, we obtain the total number of ports at the input-side as follows.

Equation (17) gives us the number of ports required at $\mathrm{FXC}$ layer at each node. After switching at the FXC layer, the number of remaining lightpaths going through the $\mathrm{BXC}$ layer is $M P-\left(F_{b}+F_{d}+F_{a}\right) \times \mathcal{K} \times \delta \times N$ Thus, we have:

$$
\text { Total }=F X C_{n} \times N+\frac{M P-\left(F_{b}+F_{d}+F_{a}\right) \times \mathcal{K} \times \delta \times N}{\mathcal{W}}
$$

\section{Upper and Lower bounds}

Note that, in the best case, all the $G+A$ lightpaths can be added/switched only at the FXC layer (i.e., no need for $\mathrm{WXC} / \mathrm{BXC}$ ), and hence the lower bound on the number of ports needed at each node is $\left(\left\lceil\frac{A}{\mathcal{K}}\right\rceil+\left\lceil\frac{G}{\mathcal{K}}\right\rceil\right) \times \delta$ FXC ports. On the other hand, in the worst case, all these lightpaths will have to be added/switched at the WXC layer, and thus the maximum $W X C_{n}$ is $(G+A) \times \delta$. At the FXC layer, the maximum number of ports needed at each node should also be bounded by $(G+A) \times \delta$. In addition, in the worst case, all the input fibers may go through the FTB demultiplexers. Thus, $B T F=$ $F T B \leq \mathcal{F} \times \delta$, and then, $F X C_{n} \leq \mathcal{F} \times \delta \times 2$. In other words, the maximum $F X C_{n}$ is $\min [(G+A) \times \delta, \mathcal{F} \times \delta \times 2]$. Similarly, the maximum $B X C_{n}$ is $\min [(G+A) \times \delta, \mathcal{F} \times \mathcal{B} \times \delta \times 2]$. Thus, the bounds for the total number of ports in the network are as follows:

$$
\begin{aligned}
\text { LowerBound }= & \left(\left\lceil\frac{A}{\mathcal{K}}\right\rceil+\left\lceil\frac{G}{\mathcal{K}}\right\rceil\right) \times \delta \times N \\
\text { UpperBound }= & \{\min [(A+G) \times \delta, \mathcal{F} \times \delta \times 2]+\min [(A+G) \\
& \times \delta, \mathcal{F} \times \mathcal{B} \times \delta \times 2]+\delta \times(A+G)\} \times N
\end{aligned}
$$

\section{NumericAl RESUlts AND Discussions}

In this section, we focus on simulation to compare WBS algorithms based on the ILP model, WBO-RWA and BPHT. We will first present the results for ILP, WBO-RWA and BPHT for a random 6-node network, as our experiments show that the optimal WBS based on ILP formulation is feasible only for such a small network. We then compare the heuristic algorithms such as BPHT, and WBO-RWA only, for the larger 14-node NSF network.

We define the following three performance-metrics. Each metric is a function of a WBS algorithm.

- Total port number ratio T(a):

$\frac{\text { Total }\left(F X C_{n}+B X C_{n}+W X C_{n}\right) \text { used by WBS algorithm' } a^{\prime}}{\text { Total }\left(O X C_{n}\right) \text { of ordinary } O X C}$ Total $\left(O X C_{n}\right)$ of ordinary $-O X C$

- Max port number ratio $\mathrm{M}(\mathbf{a})$ : $\max \left(F X C_{n}+B X C_{n}+W X C_{n}\right)$ used by WBS algorithm ' $a^{\prime}$ $\max \left(O X C_{n}\right)$ of ordinary $-O X C$

- Used wavelength-hop ratio $W(a):^{2}$ wavelength-hops used by WBS algorithm' a $^{\prime}$

The results presented below are obtained via extensive simulation, and each point is the averaged result over a large number of simulation runs with different random traffic patterns.

\section{A. Six-node Network}

For the six-node network, a traffic matrix is randomly generated, such that the number of lightpaths requested by a $(s, d)$ pair is in the range of $0 \sim 4$. When using ILP formulation, we set $\alpha=\beta=\gamma=1$ in the objective equation (1) and use CPLEX to obtain the optimal results.

For three different representative random traffic patterns where the total lightpaths (i.e. $\sum t_{p}$ ) is 25,31 and 53 respectively, Table I shows the number of ports and performance ratios for optimal WBS (based on ILP), WBO-RWA and BPHT. As the basis for the comparison, the last row (OXC) indicates the minimum total number of ports required when ordinary OXCs without WBS are used. The rows T(a), M(a) and $\mathrm{W}(\mathrm{a})$ represent the performance ratios.

TABLE I

RESULTS FOR THE SIX-NODE NETWORK $(\mathcal{F}=2, \mathcal{B}=2, \mathcal{W}=2)$

\begin{tabular}{|c|c|c|c|c|c|c|c|c|c|}
\hline & \multicolumn{3}{|c|}{ Optimal WBS } & \multicolumn{3}{|c|}{ WBO-RWA } & \multicolumn{3}{|c|}{ BPHT } \\
\hline$\sum t_{p}$ & 25 & 31 & 53 & 25 & 31 & 53 & 25 & 31 & 53 \\
\hline $\mathrm{T}(\mathrm{a})$ & 0.48 & 0.42 & 0.51 & 1.23 & 0.84 & 1.26 & 0.54 & 0.43 & 0.56 \\
\hline $\mathrm{M}(\mathrm{a})$ & 0.69 & 0.50 & 0.73 & 1.44 & 1.19 & 1.50 & 0.63 & 0.50 & 0.69 \\
\hline $\mathrm{W}(\mathrm{a})$ & 1.02 & 1.02 & 1.01 & 1.00 & 1.00 & 1.00 & 1.00 & 1.02 & 1.02 \\
\hline OXC & 71 & $\left(\sum t_{p}=25\right) ;$ & 83 & $\left(\sum t_{p}=31\right) ;$ & 142 & $\left(\sum t_{p}=53\right)$ \\
\hline
\end{tabular}

From the table, we see that the performance of BPHT is close to that of the ILP model (Optimal WBS) and much better than that of WBO-RWA, in particular, BPHT can save about $50 \%$ of the total ports than using just ordinary OXCs. In addition, in the process of trying to reduce the total number of ports, both our ILP solution and heuristic (BPHT) have

\footnotetext{
${ }^{2}$ Note that by definition $\mathrm{W}(\mathrm{WBO}-\mathrm{RWA})=1$.
} 
$\mathrm{W}(\mathrm{a})>1$, that is, use more wavelength-hop (WH) than the ILP solution for RWA (i.e. WBO-RWA). This can be explained as follows: sometimes, to reduce port count, a longer path that utilizes a wavelength in a band may be chosen even though a shorter path (that cannot be packed into a band) exists. In other words, minimizing the number of ports at MG-OXC does not necessarily imply minimizing the number of WHs (even though minimizing WHs in networks without MG-OXC is equivalent to minimizing the number of ports). In fact, there is a trade-off between the required number of WHs and ports.

Though the results are not shown, we note that in multi-fiber networks there is a slight improvement in the performance in terms of $\mathrm{T}(\mathrm{a}), \mathrm{M}(\mathrm{a})$ and $\mathrm{W}(\mathrm{a})$ (over single-fiber networks), which we attribute to the inherent partial wavelength conversion capability. Additional results for BPHT and WBO-RWA in single and multiple fiber networks will be shown in Section VI-C.

For a large network such as the NSF network, the ILP becomes intractable, hence we will only study the previously described heuristic algorithms in the following sections.

\section{B. NSF network - Uniform Traffic}

In this section, we focus on the validation of our analysis of BPHT (Section V). Figure 4 shows the number of ports

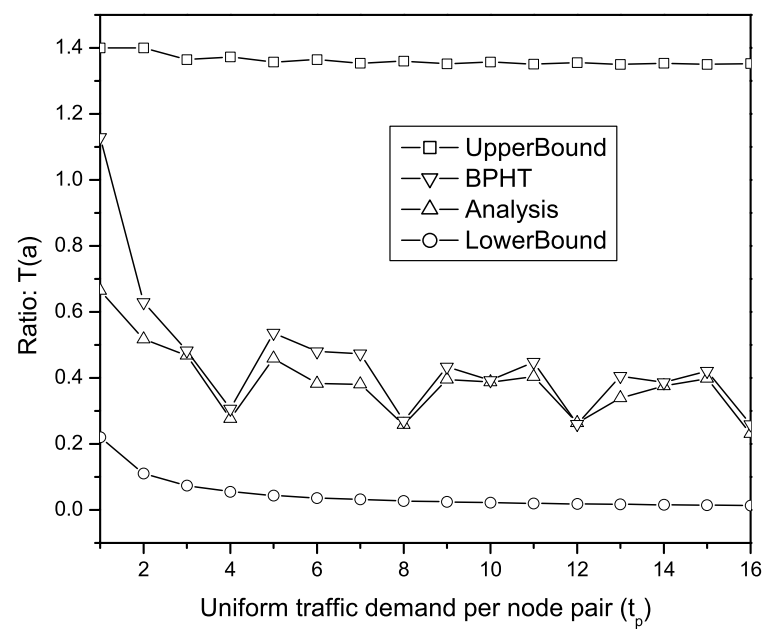

Fig. 4. $\mathrm{T}(\mathrm{a})$ in NSF network $(\mathcal{F}=2)$.

obtained from our analysis with simulation results for the NSF network assuming uniform traffic. Curve "Analysis" is from Equation (20) and (21), curve "LowerBound" is from Equation (22) and curve "UpperBound" is from Equation (23). The figure illustrate how $\mathrm{T}(\mathrm{a})$ changes with the traffic intensity $t_{p}$ (traffic demand per node pair), assuming that each fiber has 30 bands, and each band has 4 wavelengths (so the total number of wavelengths, is $\mathcal{F} \times \mathcal{B} \times \mathcal{W}=240$ ).

We see that when using heuristic algorithm BPHT, the number of MG-OXC ports drops significantly when $t_{p}$ is a multiple of $\mathcal{W}=4$ (e.g. $t_{p}$ is $4,8,12,16$ etc.), the number of wavelengths in a band. This can be explained as in Figure 5 (a), when the traffic demand (number of lightpaths) per node pair is a multiple of the number of wavelengths in a band (i.e. $\mathcal{W}$ ), using algorithm BPHT will enable MG-OXCs to add/drop/bypass traffic at the band granularity, rather than the wavelength granularity. This is similar to having an ordinaryOXC, wherein each port switches a band of wavelengths, thus reducing the number of ports. Further, note that when $t_{p}$ is such that $\operatorname{Mod}\left(t_{p}, 4\right)=2$ (e.g. $t_{p}$ is 2,6,10,14 etc.), the performance of BPHT is again close to its analytical value, this is due to the high probability of grouping bypass traffic as in Figure 5(b).

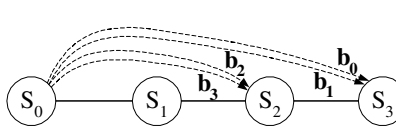

(a)Uniform traffic $\left(t_{\mathrm{p}}=8\right)$

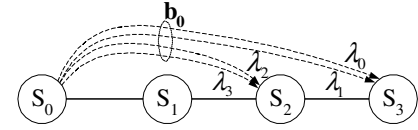

(b)Uniform traffic $\left(\mathrm{t}_{\mathrm{p}}=2\right)$
Fig. 5. An example of band grouping by BPHT $(\mathcal{W}=4)$

We can also see that the performance analysis is accurate, as verified by our simulation results, especially at the points where the traffic demand is a multiple of the waveband granularity. At other points, the assumption in the analysis such as every add/drop traffic is evenly distributed on all the output links causes the deviation in the performance of BPHT from the analytical results.

\section{NSF Network - Non-uniform Traffic}

In our experiments with multi-fiber networks, we once again find that BPHT performs better than its variations, and hence only show the comparison of algorithms BPHT and WBO-RWA, for the sake of conciseness.

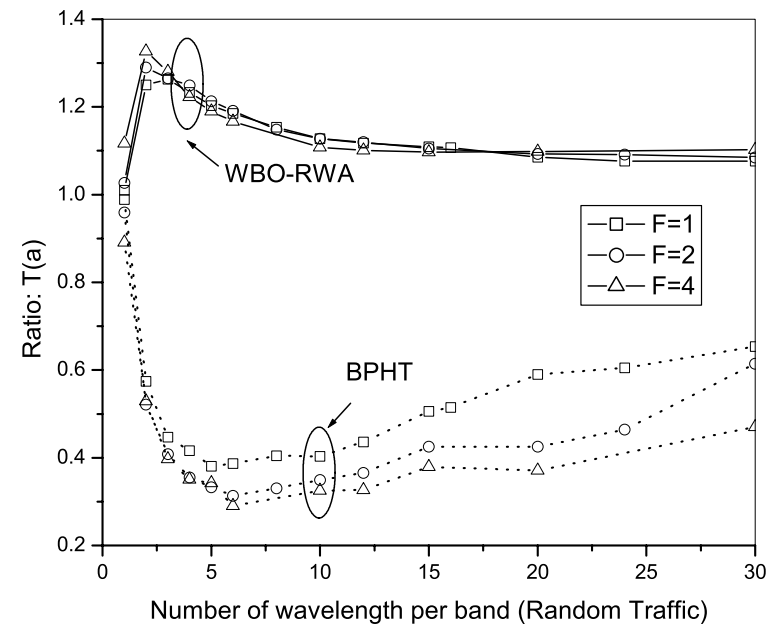

Fig. 6. Ratio T(a) (Fixed Load) 


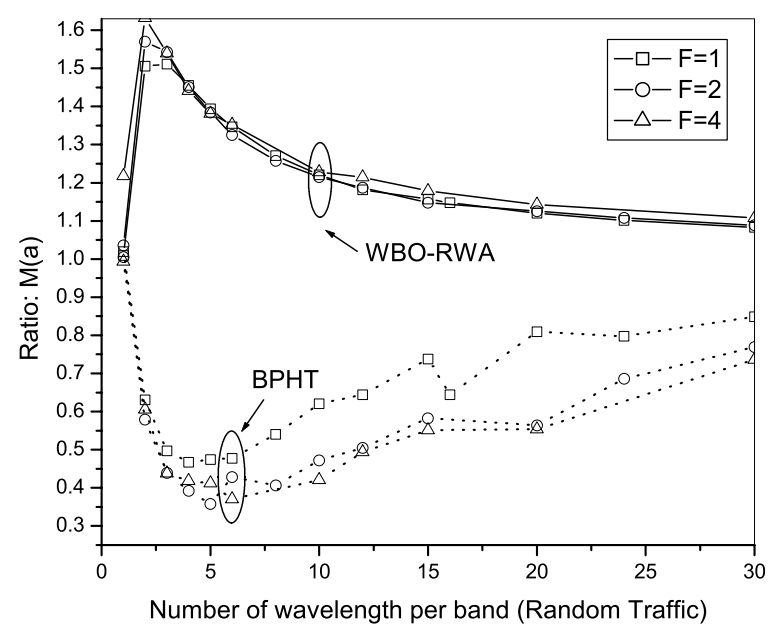

Fig. 7. Ratio M(a) (Fixed Load)

1) Fixed Load: Figures 6 and 7 illustrate how the ratios $T(a)$ and $M(a)$ vary with changing waveband granularity (i.e. number of wavelengths in a band) and number of fibers but a fixed number of total wavelengths per link (i.e. $\mathcal{F} \times \mathcal{B} \times \mathcal{W}=$ 240) and a fixed traffic load (i.e. the total traffic does not change with $\mathcal{F}$ or $\mathcal{B}$ or $\mathcal{W}$ ) but a random pattern. From the figures, we notice that the total number of ports in the network and the maximum number of ports at a node among all nodes by using BPHT is much less than those from WBO-RWA, and heuristic WBO-RWA requires more ports at MG-OXC than using ordinary OXCs (as T(WBO-RWA) $>1$ ). Interestingly, the curves for BPHT in Figures 6 and 7 also indicate that with an appropriate waveband granularity $(\mathcal{W} \simeq 6$ ), BPHT performs the best in terms of both $T(a)$ and $M(a)$, achieving a savings of nearly $70 \%$ in number of ports when using MGOXCs instead of ordinary OXCs.

More specifically, we notice that multi-fiber MG-OXC networks perform better than single-fiber MG-OXC networks, as they can achieve a larger reduction in port count when using BPHT. This is because with multiple fibers (e.g. $\mathcal{F}=4$ ) there is a higher probability to switch lightpaths as a group (whole fiber or band). In single-fiber networks the advantage of having a FXC layer and fiber switching is not evident [1]. On the other hand, the situation is slightly reversed for WBO-RWA, since WBO-RWA does not appropriately consider band or fiber switching, the wavelength assignment is done in manner unsuitable for reducing port count. Hence the benefit of multifiber in reducing port count does not show up in WBO-RWA algorithm.

In addition, Figure 8 shows how the ratios $T(a), W(a)$ and $M(a)$ vary with changing number of fibers per link (i.e. $\mathcal{F}$ ) but a fixed number of wavelengths per link (i.e. $\mathcal{F} \times \mathcal{B} \times \mathcal{W}=240$ ), a fixed waveband granularity (i.e. $\mathcal{W}=4$ ) and fixed traffic load. From the figure we can see that with appropriate number of fibers, having multiple fibers per link reduces necessary ports when compared to having only a single fiber. On the other hand, having too many fibers causes an increase in port count. The ratio $W(B P H T)$, does not vary much with a change in the number of fibers, but remains larger than 1 by a small amount indicating the trade-off between the number of WHs and ports.

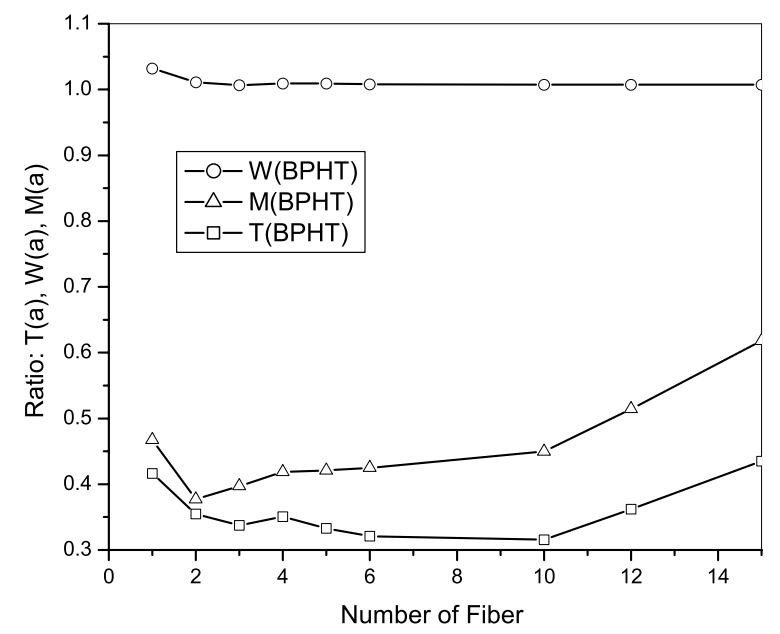

Fig. 8. Effect of multiple fibers per link (Fixed Load)

2) Proportional Load: Figures 9 - 10 depict the variation in total port number and maximum port number with changing waveband granularity and fiber number but with a fixed number of wavelengths per fiber (i.e. $\mathcal{W} \times \mathcal{B}=60$ ).

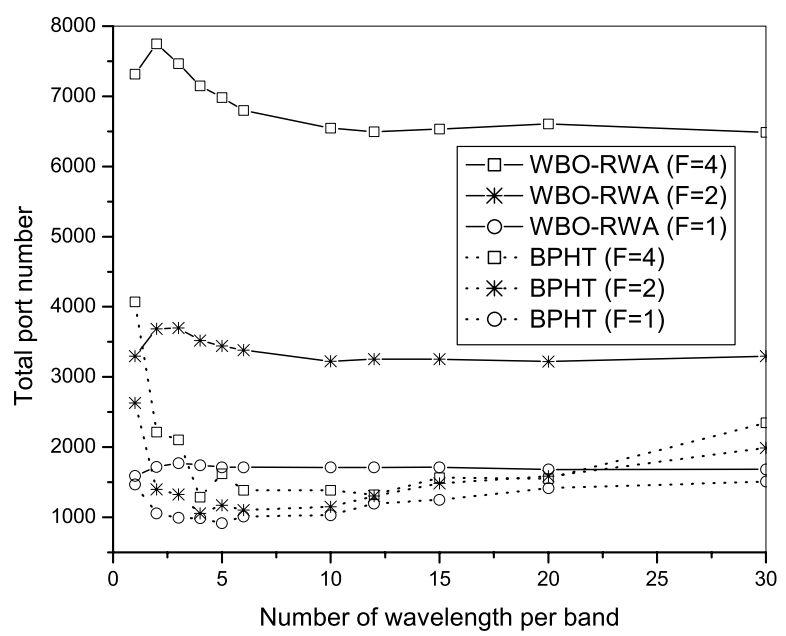

Fig. 9. Total port count (Proportional Load)

The traffic demand is directly proportional to the number of fibers (i.e. if $\mathcal{F}$ is doubled, the traffic demand $T[p]$ is also doubled). Although the results of $\mathrm{W}(\mathrm{a})$ are not shown here due 


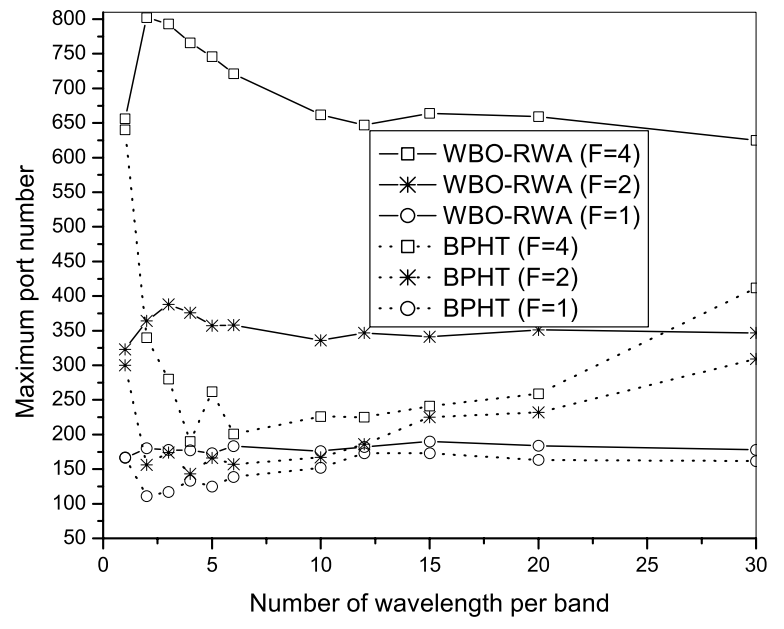

Fig. 10. Maximum number of ports (Proportional Load)

to space limitation, the WHs increase almost proportionally to the number of fibers (and traffic load) for BPHT. On the other hand, we notice that the total number of ports and maximum port number increase sub-linearly with an increase in the number of fibers (and traffic load) for BPHT. This is because there is a higher probability for wavelength grouping (into fibers and bands) in multi-fiber networks using the BPHT algorithm. However, we find that the total port number and maximum port number for WBO-RWA increase rapidly with an increase in the number of fibers, further indicating the effectiveness of our proposed BPHT algorithm.

\section{CONCLUSION}

In this work, we have studied the problem of optimal WBS in multi-granular all-optical mesh networks. We have proposed a new MG-OXC architecture which is more flexible than any existing architectures. We have also adopted the most powerful waveband assignment strategy and developed a corresponding ILP and an efficient heuristic algorithm called BPHT. The ILP model and the heuristic algorithm (which uses a new multifiber waveband assignment algorithm) can handle the case with multiple fibers per link, and hence are also more general than our previously proposed single-fiber solutions.

We have verified that the proposed BPHT heuristic can achieve near-optimal results by comparing its performance with that of the ILP formulation (which is feasible for small networks only). In addition, the performance of BPHT has been analyzed and the results from analysis are verified with simulation. We have also compared the performance of BPHT with that of a heuristic that uses ILP to perform optimal RWA via extensive simulations for varying network topologies and traffic patterns, and shown that BPHT is significantly better.

Our performance evaluation has also shown that WBS is even more beneficial in multi-fiber networks. In addition, the waveband granularity has a large effect on the performance of WBS networks. In particular, with appropriate waveband granularity, using MG-OXCs in multi-fiber networks can save up to $70 \%$ ports compared to using ordinary-OXCs. While our ILP formulations and heuristics are especially useful for the efficient design of MG-OXC nodes (i.e. the dimensioning of the switching matrices) for a given set of traffic demands, they can also be used to minimize the number of used active ports in an existing network, and thus lower network operating costs, and reduce blocking probability of future requests.

\section{REFERENCES}

[1] X. Cao, Y. Xiong, V. Anand, and C. Qiao, "Wavelength band switching in multi-granular all-optical networks," in SPIE's Proc. vol. 4874, OptiComm'02, Boston Massachusetts, 2002, pp. 198-210.

[2] K. Harada, K. Shimizu, T. Kudou, and T. Ozeki, "Hierarchical optical path cross-connect systems for large scale WDM networks," in Proceedings - OFC, 1999, pp. WM55-3.

[3] L. Noirie, M. Vigoureux, and E. Dotaro, "Impact of intermediate grouping on the dimensioning of multi-granularity optical networks," in Proceedings - OFC, 2001, pp. TuG3-3.

[4] E.Dotaro, D. Papadimitriou, L. Ciavaglia, M. Vigoureux, R. Douville, and L. Noirie, "Optical multi-granularity architectural framework," draftdotaro-ipo-multi-granularity-01.txt, Nov. 2001.

[5] A. A. M. Saleh and J. M. Simmons, "Architectural principles of optical regional and metropolitan access networks," J. of Lightwave Technology, vol. 17, no. 12, pp. 2431-2448, Dec. 1999.

[6] O. Gerstel, R. Ramaswami, and W.-K. Wang, "Making use of a two stage multiplexing scheme in a WDM network," in Proceedings - OFC, 2000, pp. ThD1-3.

[7] R. Izmailov, S. Ganguly, Y. Suemura, I. Nishioka, Y. Maeno, and $\mathrm{S}$. Araki, "Waveband routing in optical networks," in IEEE International Conference on Communications (ICC'02), New York, 2002.

[8] L. Noirie, F. Dorgeuille, and A. Bisson, "32 × $10 \mathrm{gbit} / \mathrm{s}$ DWDM metropolitan network demonstration with 10 waveband- adms and 155 km teralight metro fiber," in Proceedings - OFC, 2002, p. ThH4.

[9] R. Lingampalli and P. Vengalam, "Effect of wavelength and waveband grooming on all-optical networks with single layer photonic switching," in Proceedings - OFC, 2002, p. ThP4.

[10] M. Lee, J. Yu, Y. Kim, C. Kang, and J. Park, "Design of hierarchical crossconnect WDM networks employing a two-stage multiplexing scheme of waveband and wavelength," in IEEE JSAC, Vol. 20, No. 1, Jan. 2002, pp. 166-171.

[11] D. Banerjee and B. Mukherjee, "A practical approach for routing and wavelength assignment in large wavelength-routed optical networks," IEEE JSAC, vol. 14, pp. 903-908, June 1996.

[12] B. Mukherjee, D. Banerjee, S. Ramamurthy and A. Mukherjee, "Some principles for designing a wide-area optical network," IEEE/ACM Tran. on Networking, vol. 4, pp. 684-696, Oct. 1996.

[13] I. Chlamtac, A. Ganz and G. Karmia, "Lightpath communications: An approach to High Bandwidth Optical WAN's," IEEE Tran. on Comm., vol. 40, no. 2, pp. 1171-1182, July 1992.

[14] A. Mokhtar and M. Azizoglu, "Adaptive Wavelength Routing in AllOptical Networks," IEEE/ACM Tran. on Networking, vol. 6, no. 2, pp. 197-206, April 1998.

[15] R. Ramaswami and G. H. Sasaki, "Multiwavelength optical networks with limited wavelength conversion,' in Proceedings of INFOCOM '97, 1997, pp. 490-499.

[16] G. Jeong and E. Ayanoglu, "Comparison of wavelength-interchanging and wavelength-selective cross-connects in multiwavelength all-optical networks," in Proceedings of INFOCOM '96, 1996, pp. 156-163.

[17] R. Sabella, E. Iannone, M. Listani, M. Berdusco, and S. Binetti, "Impact of transmission performance on path routing in all-optical transport networks," in J. of Lightwave Technology vol. 16, No. 11, Nov. 1998, pp. 1965-1972.

[18] J. Yen, "Finding the k shortest loopless paths in a network," in Management Science, Vol.17, No.11, 1971, pp. 712-16. 